\title{
IDENTIFICACIÓN DE SITIOS CON POTENCIAL PARA LA DISPOSICIÓN FINAL DE RESIDUOS SÓLIDOS URBANOS EN EL MUNICIPIO DE TEPIC, NAYARIT, MÉXICO
}

\author{
Identification of potential sites for urban solid waste disposal in the municipality of Tepic, Nayarit, Mexico
}

\author{
Claudia E. SALDAÑA DURÁN* y Oyolsi NÁJERA GONZÁLEZ
}

Universidad Autónoma de Nayarit, Ciudad de la Cultura s/n, Col. Centro, 63000 Tepic, Nayarit, México

*Autora para correspondencia; cesduran@uan.edu.mx

(Recibido julio 2018; aceptado marzo 2019)

Palabras clave: sistemas de información geográfica, sostenibilidad, relleno sanitario

\section{RESUMEN}

El crecimiento de la población en áreas urbanas y el desarrollo de las ciudades debe apoyar la sostenibilidad ambiental en los sistemas de manejo de residuos sólidos. La disposición final de estos residuos es un problema serio, ya que es el punto crítico para el control de la contaminación ambiental de suelos y fuentes de agua locales. Por lo anterior, la necesidad de designar sitios adecuados para su disposición final es evidente y justificada. En este contexto, el objetivo de este trabajo es identificar zonas potenciales para la ubicación de un relleno sanitario de residuos sólidos en el municipio de Tepic. Se realizó un análisis espacial del municipio contrastando cuatro criterios señalados en la Norma Oficial Mexicana NOM-083-SEMARNAT-2003, dos rasgos naturales más, pendiente y cobertura, y uso del terreno. Se logró identificar un $5.4 \%$ de superficie del municipio de Tepic (cerca de 9090.8 ha) con rasgos apropiados para la ubicación de un relleno sanitario; el $94.6 \%$ restante presenta al menos una característica que lo limita o restringe para este fin. Este trabajo representa un primer acercamiento para la identificación de áreas adecuadas para los sitios de disposición final de residuos sólidos urbanos.

Key words: geographic information systems, sustainability, landfills

\begin{abstract}
The growth of population in urban areas and the development of cities should support environmental sustainability in solid waste management systems. The final disposal of this waste is a serious problem, since it is the critical point for the control of environmental contamination of local soils and water sources. Therefore, the need to designate suitable sites for their final disposal is evident and justified. In this context, the objective of this paper is to identify potential areas for the location of a solid waste landfill in the municipality of Tepic. A spatial analysis of the municipality was carried out, contrasting four criteria indicated in the Official Mexican Standard NOM-083-SEMARNAT-2003, two other natural features, slope and coverage, andland use. It has been possible to identify $5.4 \%$ of the surface of the municipality of Tepic (about $9090.8 \mathrm{ha}$ ) with appropriate land features for the location of a sanitary landfill; the remaining $94.6 \%$ presents at least one characteristic that limits or restricts this purpose. This work represents a first approach for the identification of suitable areas for final disposal sites of urban solid waste.
\end{abstract}




\section{INTRODUCCIÓN}

En un mundo cada vez más urbano y degradado ambientalmente, se plantea que cerca del 60 al $75 \%$ de la población mundial viva en zonas urbanas para el periodo 2025 al 2050 (UNDESA 2014)technical, legal, social or environmental issues. The decisionmaking process includes the identification of six candidate MSW plant sites and 21 criteria grouped into clusters for the construction of a network. Two technicians of the Metropolitan Waste Disposal Agency acted as decision makers (DMs. Este planteamiento conduce a muchos problemas en el entorno urbano, como concentración de población, escasez de vivienda y recursos, reducción de la biodiversidad, contaminación del aire, suelo y agua (Khan y Samadder 2014).

La disposición final de residuos es un problema grave, ya que es el punto crítico para el control de la contaminación ambiental de suelos y fuentes de agua locales. En el pasado reciente, e incluso en la actualidad, un gran número de ciudades mexicanas han eliminado sus residuos municipales de forma inapropiada, utilizando vertederos no controlados para enterrar su basura, ocasionando una cadena de degradación ambiental. La gestión de los residuos sólidos se define como la disciplina asociada al control de la generación, almacenamiento, recolección, transferencia y transporte, procesamiento y evacuación de residuos sólidos de forma que armonice con los mejores principios de la salud pública, la economía, la ingeniería, la conservación, la estética y otras consideraciones ambientales y públicas (Vergara y Tchobanoglous 2012). Dentro de este ámbito se incluyen todas las funciones administrativas, financieras, legales, de planificación y de ingeniería.

La evaluación de un nuevo sitio para la disposición final de residuos es una tarea complicada, ya que requiere del conocimiento de diversos campos sociales y ambientales, como la ciencia del suelo, la ingeniería, hidrogeología, geología, topografía, uso del suelo, sociología y economía. La Norma Oficial Mexicana NOM-083-SEMARNAT-2003 (SEMARNAT 2004) establece especificaciones de protección ambiental para la selección del sitio, diseño, construcción, operación, monitoreo, clausura y obras complementarias de un sitio de disposición final de residuos sólidos urbanos y de manejo especial. Define parámetros como distancia a carreteras, habitación, elementos clave de infraestructura y propensión del suelo a lixiviar contaminantes. Por lo tanto, la selección de áreas para un relleno sanitario es un proceso complejo en el que se involucran variables reglamentarias, así como condiciones físicas, ambientales, económicas y de salud, además de impactos socioculturales (Sadek et al. 2006).

Por lo anterior, es necesario analizar los criterios de la NOM-083-SEMARNAT-2003 para determinar las ubicaciones optimas de los sitios de disposición final de residuos sólidos, estaciones de transferencia, estaciones de transferencia a rellenos sanitarios, así como la planificación de rutas para vehículos que transportan residuos en la recolección y el monitoreo a largo plazo de éstos. La creciente generación de residuos sólidos en las ciudades y su disposición final es uno de los mayores retos ambientales que enfrentan las autoridades municipales.

Los sistemas de información geográfica (SIG) son una herramienta útil en la selección de sitios adecuados para la ubicación de rellenos sanitarios municipales, a partir de información ambiental como geología, hidrogeología, uso del suelo y distancia hacia asentamientos, cuerpos de agua superficiales, áreas protegidas (ecológicas, científicas o históricas) y criterios económicos como carreteras, pendientes y elevaciones (Zamorano et al. 2008; Guiqin et al. 2009; Khan y Samadder 2014; Chabuk et al. 2016) which is based on EVIAVE, a landfill diagnosis method developed at the University of Granada. Geographical Information Systems (GIS. El potencial de los SIG reduce el tiempo y el costo en la selección del sitio y proporciona un banco de datos digital que facilita el manejo y la correlación de grandes cantidades de datos complejos a través de la visualización de la representación gráfica. Esto permite a los responsables de las políticas enlazar fuentes de información dispares, realizar análisis, y visualizar tanto tendencias como resultados del proyecto, estrategias y objetivos de planificación a largo plazo (Malczewski 2004).

Los SIG se han convertido en una importante herramienta para el análisis espacial enfocado a la sustentabilidad para los usos del suelo. Se han realizado estudios sobre residuos sólidos urbanos para identificar sitios de disposición final aplicando SIG (Chabuk et al. 2016; El Maguiri et al. 2016; Güler y Yomralıoğlu 2017).

En México, una de las prácticas más comunes para la disposición final de los residuos sólidos es el tiradero a cielo abierto o relleno sanitario, el cual se desarrolla al margen de la normatividad vigente (NOM-083-SEMARNAT-2003). Con este esquema de manejo y disposición, los residuos urbanos que se depositan en barrancas o predios (generalmente en los alrededores de centros de población) se manejan de manera inadecuada, improvisando y operando 
basureros en sitios inapropiados, sin infraestructura para su manejo (Bernache 2015), lo que contribuye a la generación de problemas sanitarios y ambientales.

Con base en su contexto geográfico-administrativo, se establece que la ciudad de Tepic, Nayarit, México, es una urbe intermedia, con una población de 413608 habitantes (INEGI 2016). El manejo de residuos sólidos urbanos (RSU) en este centro urbano representa un problema para la gestión municipal de los servicios públicos. Los montos de generación de residuos han aumentado de $300 \mathrm{t}$ en 2003 a $600 \mathrm{t}$ en $2011,800 t$ en 2015 y se espera que aumente a 950 t en 2020 (DAP 2016). Los residuos de la ciudad se eliminan en el sitio a cielo abierto llamado El Iztete, el cual ha agotado ambientalmente su vida útil, ya que ha superado su capacidad. De esta forma, el problema de los residuos sólidos es una amenaza grave para la calidad de vida en la ciudad, de ahí la necesidad de identificar sitios adecuados para implementar la disposición final de los residuos, a fin de proteger la salud de la población y el ambiente. En este contexto, el objetivo de este trabajo es identificar zonas potenciales para la ubicación de rellenos sanitarios para disponer los residuos sólidos del municipio de Tepic.

\section{MATERIALES Y MÉTODOS}

\section{Área de estudio}

El municipio de Tepic cuenta con una actividad económica en la que predomina el sector de servicios con el $56.15 \%$, incluyendo transporte, gobierno y otros servicios; le sigue el sector comercio con el $20.24 \%$; el sector secundario ocupa el tercer lugar y comprende la minería, la extracción de petróleo y gas, industria manufacturera, electricidad, agua y construcción; y por último, el sector primario, que comprende agricultura, ganadería, silvicultura, pesca y caza. El municipio tiene una superficie de 168781 ha; una población de 429363 habitantes (INEGI 2016), y se localiza en la parte central del estado en las coordenadas métricas extremas X 484561, Y 2365545, y X 541801, Y 2417198. Colinda con los municipios de Santiago Ixcuintla y El Nayar al norte; con el municipio de Xalisco al sur; con El Nayar y Santa María del Oro al este, y al oeste con los municipios de San Blas y Santiago Ixcuintla (Fig. 1).

\section{MATERIAL Y MÉTODOS}

Para el manejo de mapas se empleó información cartográfica digital del área en estudio sobre locali-

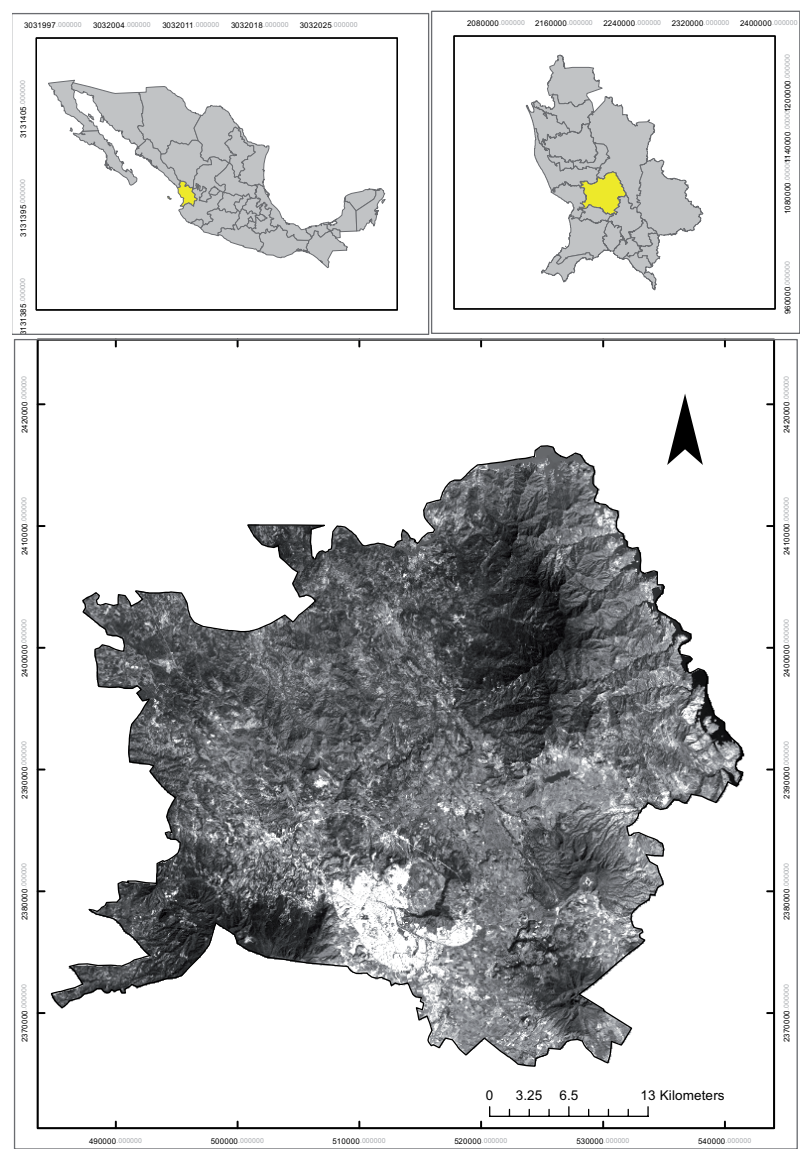

Fig. 1. Localización del área en estudio. Fuente: elaboración propia con información del INEGI. (Marco geoestadístico de Nayarit) e imagen del satélite Landsat 4

dades, topografía, hidrología superficial y cuerpos de agua, vegetación, vías de comunicación y áreas naturales protegidas, obtenida principalmente del Instituto Nacional de Estadística y Geografía (INEGI), que representa seis elementos de restricción: cuatro normativas presentes en la NOM-083-SEMARNAT-2003 y dos no normativas. Los mapas fueron proyectados sobre una imagen de satélite LandSat 4, 2016 (Proyección Elipsoide WGS84, UTM zona 13, composición bandas 432) obtenida del sitio oficial del Servicio Geológico de los Estados Unidos (USGS 2018).

\section{Delimitación del área de estudio}

Se tomó como área de estudio el límite administrativo del municipio de Tepic, empleando coordenadas UTM y proyección WGRS84 (fig. 2).

\section{Identificación de localidades involucradas}

La identificación de las poblaciones se realizó a partir de información del INEGI (2016), siguiendo los criterios de la NOM 083-SEMARNAT-2003. 


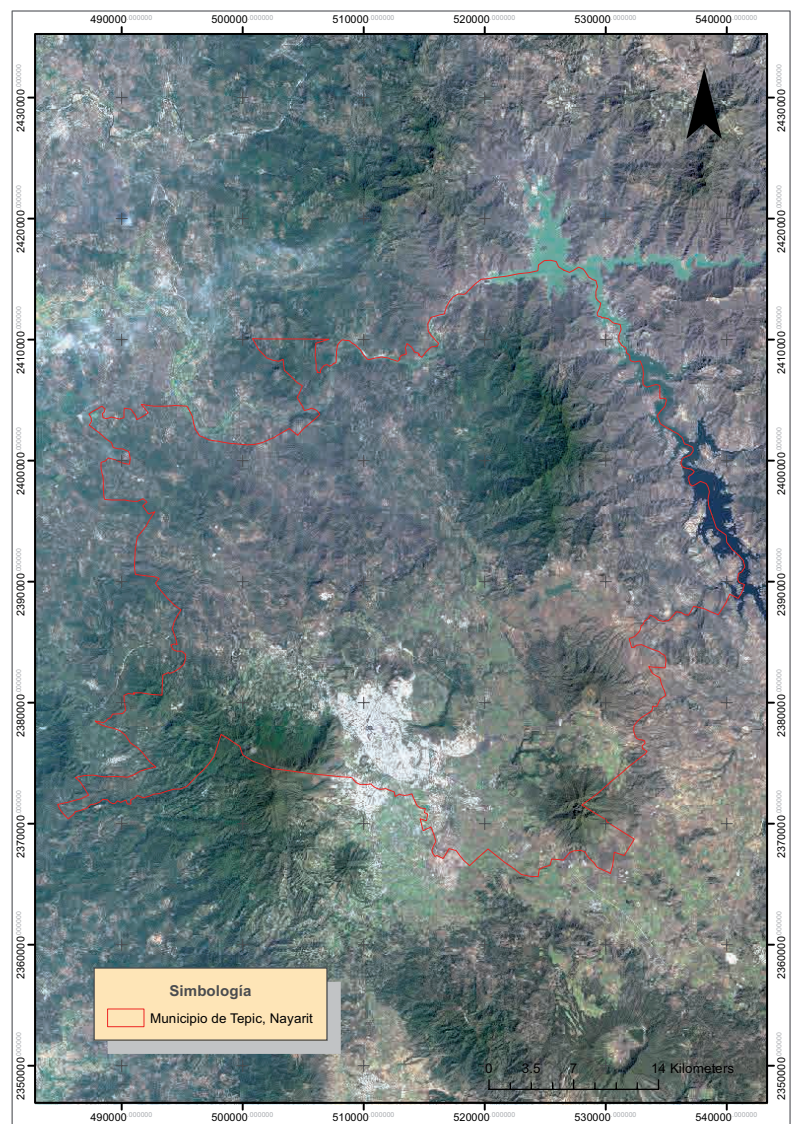

Fig. 2. Área de estudio. Fuente: elaboración propia con información del INEGI (Marco geoestadístico de Nayarit) e imagen del satélite Landsat 4

\section{Localización de infraestructura}

En este apartado se consideraron como infraestructura las vías de comunicaciones aéreas, concretamente el Aeropuerto Internacional Amado Nervo.

\section{Cauces, cuerpos de agua y superficies de inundación}

Los rasgos naturales corresponden a cauces y cuerpos de agua de flujo continuo, obtenidos de la información hidrológica del INEGI (2016).

\section{Areas naturales protegidas}

La información sobre este tema se obtuvo del Programa de Conservación y Manejo del Área Natural Protegida Reserva de la Biosfera Estatal Sierra de San Juan (SEDERMA 2008).

\section{Rasgos restrictivos no contemplados en la nor- mativa vigente}

Pendiente del terreno

Se construyó un mapa de pendientes de la zona de estudio a partir de las curvas de nivel (Segura et al.
CUADRO I. CLASIFICACIÓN DE PENDIENTES DEL TERRENO EN EL MUNICIPIO DE TEPIC, NAYARIT

\begin{tabular}{lc}
\hline Rango de pendiente (grados) & Clase de pendiente \\
\hline $0-8$ & Pendiente baja \\
$8-16$ & Moderada \\
$16-35$ & Fuerte \\
$35-90$ & Muy fuerte \\
\hline
\end{tabular}

2011), y se clasificaron en cuatro clases, excluyendo en el mapa las pendientes mayores a $35^{\circ}$ (Cuadro I). Cobertura y uso del suelo

Este criterio consideró la importancia de excluir aquellas áreas donde la ubicación de un relleno sanitario representara un impacto negativo en asociaciones vegetales. Así, las áreas de vegetación natural no consideradas como áreas naturales protegidas (bosques de pino, encino, mixtos y selvas) presentes en la zona de estudio, representaron un rasgo de exclusión por su valor ecológico y los servicios ambientales que representan.

Los seis elementos de restricción aplicados en el análisis representaron capas binarias que fueron calificadas (con base en las especificaciones para la selección del sitio señaladas en la NOM 083-SEMARNAT-2003) como no restrictivo $=0$ y restrictivo $=1$ (Cuadro II $)$.

Aplicando técnicas de sobreposición de capas se obtuvo una regionalización con unidades de mapeo donde se representan los elementos restrictivos calificados. Con apoyo de una matriz de decisión basada en la suma de elementos restrictivos de cada unidad generada, se establecieron cinco niveles de aptitud, en los cuales se definieron como potenciales las áreas (unidades) que no presentan elementos restrictivos $($ restrictivos $=0)$ y como no potenciales aquellas que presentan al menos una característica restrictiva $($ restrictivo $\neq 0)$ (Cuadro III).

\section{RESULTADOS}

\section{Localidades involucradas}

De acuerdo con el INEGI, el área de estudio municipal cuenta con una población de 429363 habitantes que representa el $35.05 \%$ de la población total del estado, la cual se asienta en 188 localidades; de éstas, 11 son mayores a 1000 habitantes y 177 menores a esa cifra. Destaca por su tamaño e importancia la ciudad de Tepic, con una superficie de 5173 ha. En conjunto, las principales localidades del municipio cubren una superficie de 6818.5 ha (INEGI 2016) (Fig. 3). 
CUADRO II. CALIFICACIÓN DE LOS RASGOS RESTRICTIVOS.

\begin{tabular}{|c|c|c|}
\hline Rasgo de restricción & Descripción & Calificación \\
\hline $\begin{array}{l}\text { Localidades } \\
\text { (distancia de } 500 \mathrm{~m} \text { al límite del } \\
\text { centro de población) }\end{array}$ & $\begin{array}{l}\text { Centros de población mayores y menores a } 1000 \text { habi- } \\
\text { tantes, así como instalaciones federales (Centro Federal } \\
\text { de Readaptación Social) }\end{array}$ & $\begin{array}{l}\text { Más de } 500 \mathrm{~m}=0 \\
\text { Menos de } 500 \mathrm{~m}=1\end{array}$ \\
\hline $\begin{array}{l}\text { Aeropistas } \\
\text { (distancia de } 13 \mathrm{~km} \text { alrededor } \\
\text { de la instalación) }\end{array}$ & $\begin{array}{l}\text { Instalaciones de vías de comunicación aéreas naciona- } \\
\text { les e internacionales y pistas aéreas }\end{array}$ & $\begin{array}{l}\text { Más de } 13 \mathrm{~km}=0 \\
\text { Menos de } 13 \mathrm{~km}=1\end{array}$ \\
\hline $\begin{array}{l}\text { Cuerpos de agua } \\
\text { (distancia de } 300 \mathrm{~m} \text { a ambos } \\
\text { lados del cauce) }\end{array}$ & $\begin{array}{l}\text { Ríos y arroyos de flujo permanentes, cuerpos de agua y } \\
\text { manantiales }\end{array}$ & $\begin{array}{l}\text { Más de } 300 \mathrm{~m}=0 \\
\text { Menos de } 300 \mathrm{~m}=1\end{array}$ \\
\hline $\begin{array}{l}\text { Áreas naturales protegidas } \\
\text { (fuera de sus límites) }\end{array}$ & Áreas naturales protegidas federales y estatales & $\begin{array}{l}\text { Fuera de límite }=0 \\
\text { Dentro de límite }=1\end{array}$ \\
\hline $\begin{array}{l}\text { Pendiente } \\
\text { (pendiente máxima de } 30^{\circ} \text { ) }\end{array}$ & $\begin{array}{l}\text { Nivel de inclinación del terreno que puede favorecer el } \\
\text { movimiento (deslizamiento) de tierra }\end{array}$ & $\begin{array}{l}\text { Más de } 30^{\circ}=0 \\
\text { Menos de } 30^{\circ}=1\end{array}$ \\
\hline $\begin{array}{l}\text { Vegetación natural } \\
\text { (fuera de asociaciones+ natu- } \\
\text { rales) }\end{array}$ & $\begin{array}{l}\text { Asociaciones de vegetación natural (derivadas de } \\
\text { procesos de sucesión) sin alteraciones o mínimas, como } \\
\text { bosques de pino, encino, mixtos; selvas (mediana y } \\
\text { baja), vegetación secundaria arbórea }\end{array}$ & $\begin{array}{l}\text { Fuera de } \\
\text { asociaciones }=0 \\
\text { Dentro de } \\
\text { asociaciones }=1\end{array}$ \\
\hline
\end{tabular}

\section{CUADRO III. MATRIZ DE DECISIÓN}

\begin{tabular}{ccc}
\hline Restrictivos por unidad & Nivel de aptitud & Potencial \\
\hline 5 & Muy baja & \\
\cline { 1 - 2 } 4 & & \\
\hline 3 & Baja & No potenciales \\
\hline 2 & Media & \\
\hline 1 & Alta & \\
\hline 0 & Muy alta & Potenciales \\
\hline
\end{tabular}

\section{Infraestructura de vías de comunicación}

El municipio de Tepic cuenta con $476 \mathrm{~km}$ de carreteras federales, estatales, autopistas y caminos rurales, así como un aeropuerto para vuelos nacionales ubicado a menos de $13 \mathrm{~km}$ al sureste de la ciudad (Fig. 4).

\section{Cauces y cuerpos de agua}

El municipio cuenta con un número importante de corrientes de agua, entre los cuales destacan los ríos Mololoa, que atraviesa el municipio, y Santiago, que representa el límite norte del área de estudio.Cuenta además con 18 pequeños ríos de corriente de agua permanente y 25 manantiales, distribuidos en la parte central del municipio (Fig. 5).

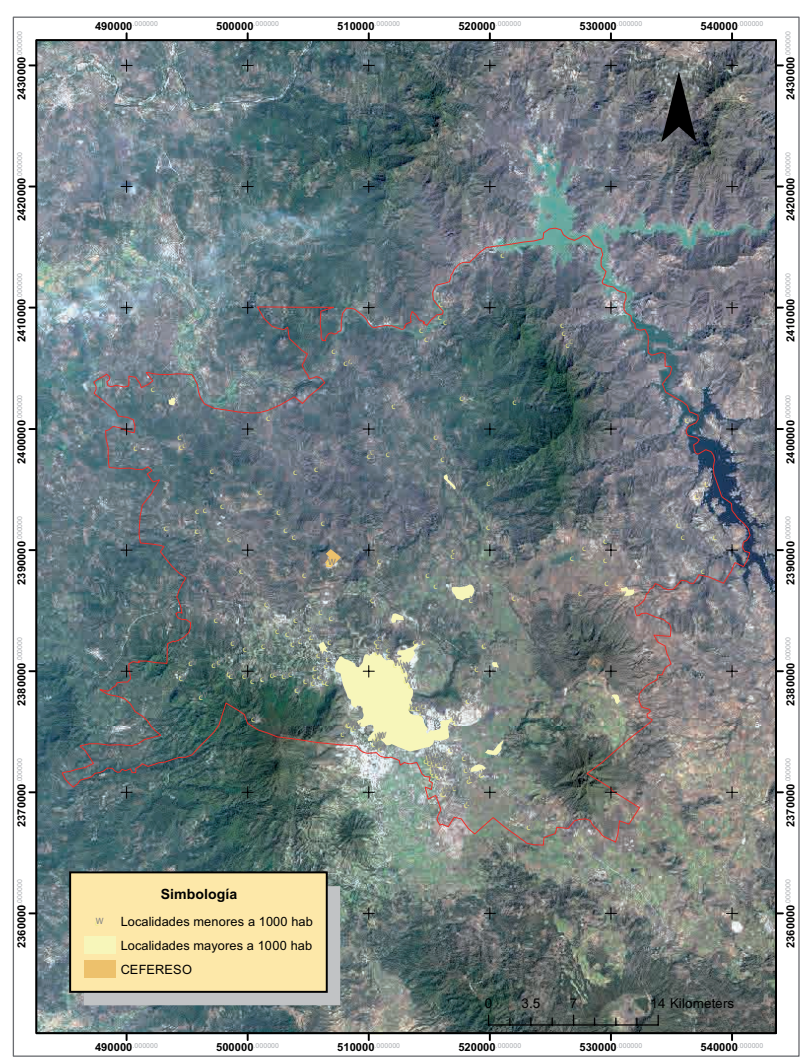

Fig. 3. Localidades involucradas. Fuente: elaboración propia con información del INEGI (Marco geoestadístico de Nayarit) e imagen del satélite Landsat 4 


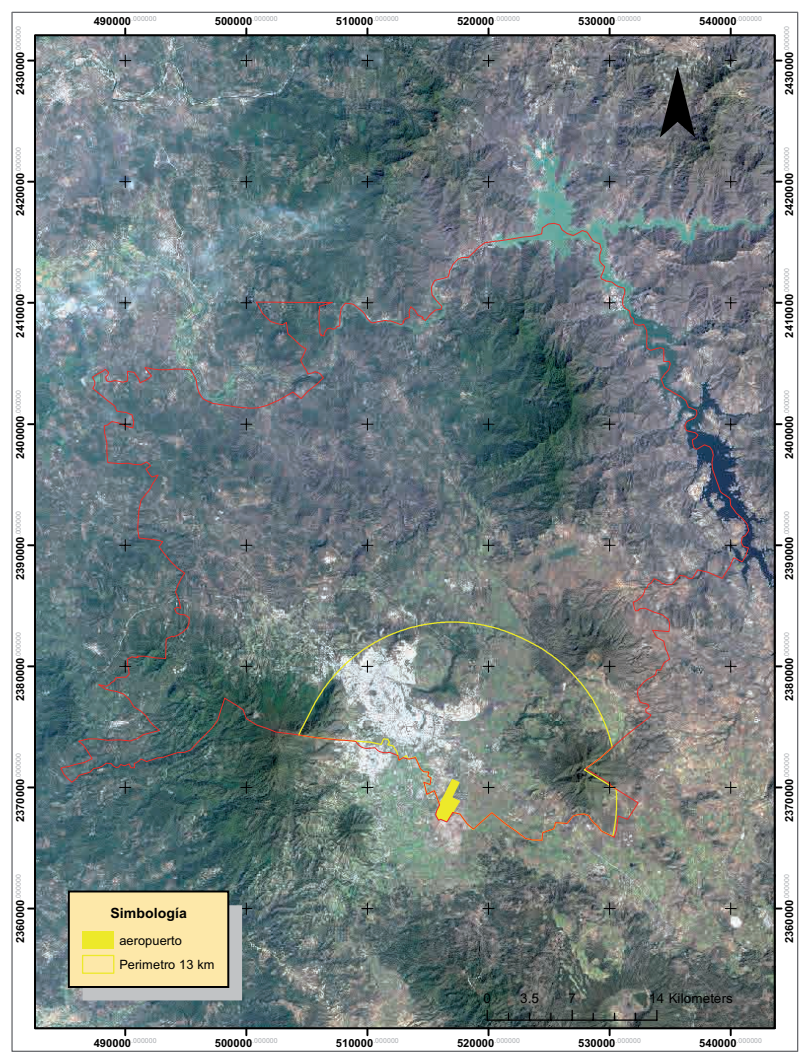

Fig. 4. Vías de comunicación. Fuente: elaboración propia con información del INEGI (Marco geoestadístico de Nayarit) e imagen del satélite Landsat 4

\section{Áreas naturales protegidas.}

El municipio cuenta con dos áreas naturales protegidas (ANP) dentro de la ciudad. La primera es el Parque Ecológico de Tepic, de carácter municipal, decretado en febrero de 2017 y con poco más de 25 ha, cuyo objetivo es conservar el humedal, incluyendo su flora y fauna, por lo que representa un refugio para diferentes especies y es fuente de bienes y servicios ambientales. La segunda es la Reserva Estatal de la Biosfera Sierra de San Juan, ANP de competencia estatal, decretada desde 1987; cuenta con una superficie de 20053 ha, de las cuales 5917.3 ha corresponden al municipio de Tepic, representando $3.5 \%$ de este territorio (INEGI 2016). Ambas áreas tienen restricciones para la instalación de un relleno sanitario (Fig. 6).

\section{Pendiente del área en estudio}

Los resultados muestran que cerca del $25 \%$ del área de estudio (42 392.5 ha) presenta pendientes de 0 a $35^{\circ}$, mientras que $74.86 \%$ (126 247.8 ha) pertenece al rango de pendientes mayores a $35^{\circ}$, lo que indica que no son adecuadas para situar un

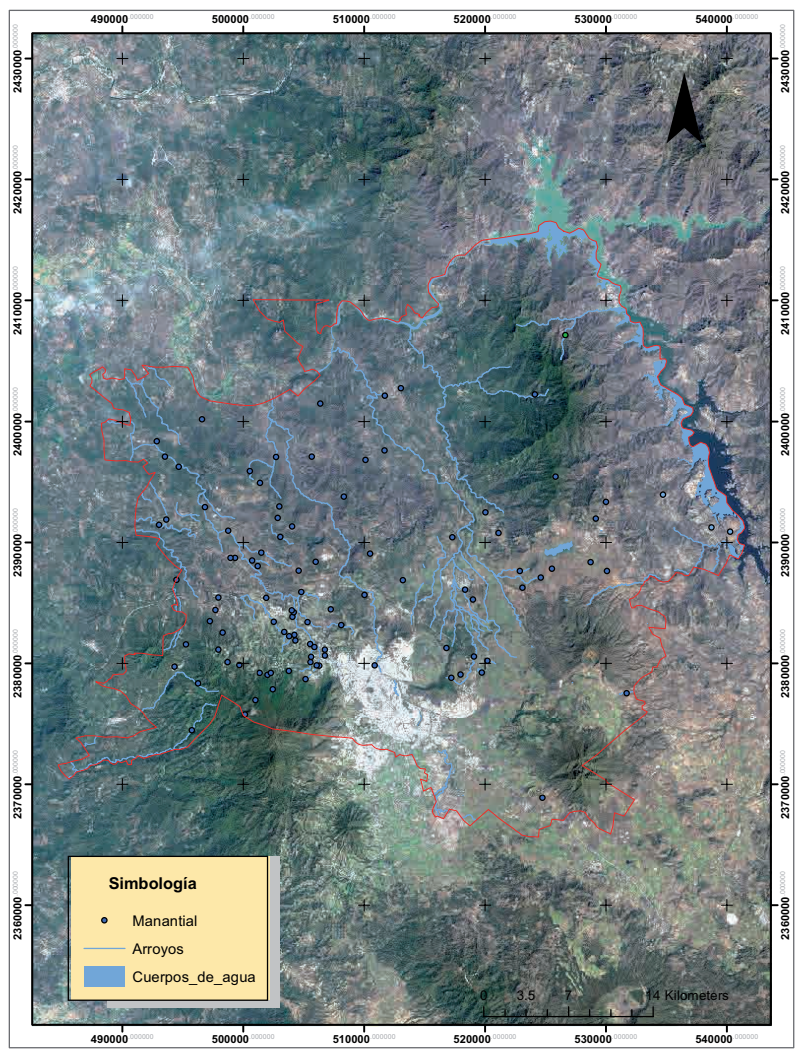

Fig. 5. Cauces y cuerpos de agua. Fuente: elaboración propia con información del INEGI (Conjunto de datos vectoriales, cartas F13 d11, d21, d31, d22, c29 y c39) e imagen del satélite Landsat 4

relleno sanitario. Este rasgo natural se relaciona con la conservación de suelos, por lo que las áreas de menor pendiente pueden considerarse adecuadas para la posible ubicación de un sitio, aunque según la normativa la pendiente de un lugar no es un rasgo restrictivo para la construcción de rellenos. En este estudio se consideraron como no adecuadas para ubicación de un relleno sanitario las pendientes mayores a $35^{\circ}$ (Fig. 7$)$.

\section{Cobertura y uso del suelo}

El uso del suelo y la cobertura vegetal del municipio de Tepic se clasifican en siete tipos, como se muestra en el cuadro IV.

Las clases "Bosques" y "Selva" restringen el área para el fin deseado en un $28.69 \%$. La clase "Bosque" (16.44 \%) comprende pinos y encinos, así como pequeños manchones de bosque mesófilo de montaña, mientras que la clase "Selva" (12.25 \%) incluye asociaciones vegetales de selva mediana y selva baja, distribuidas en las áreas de baja altitud del municipio. 


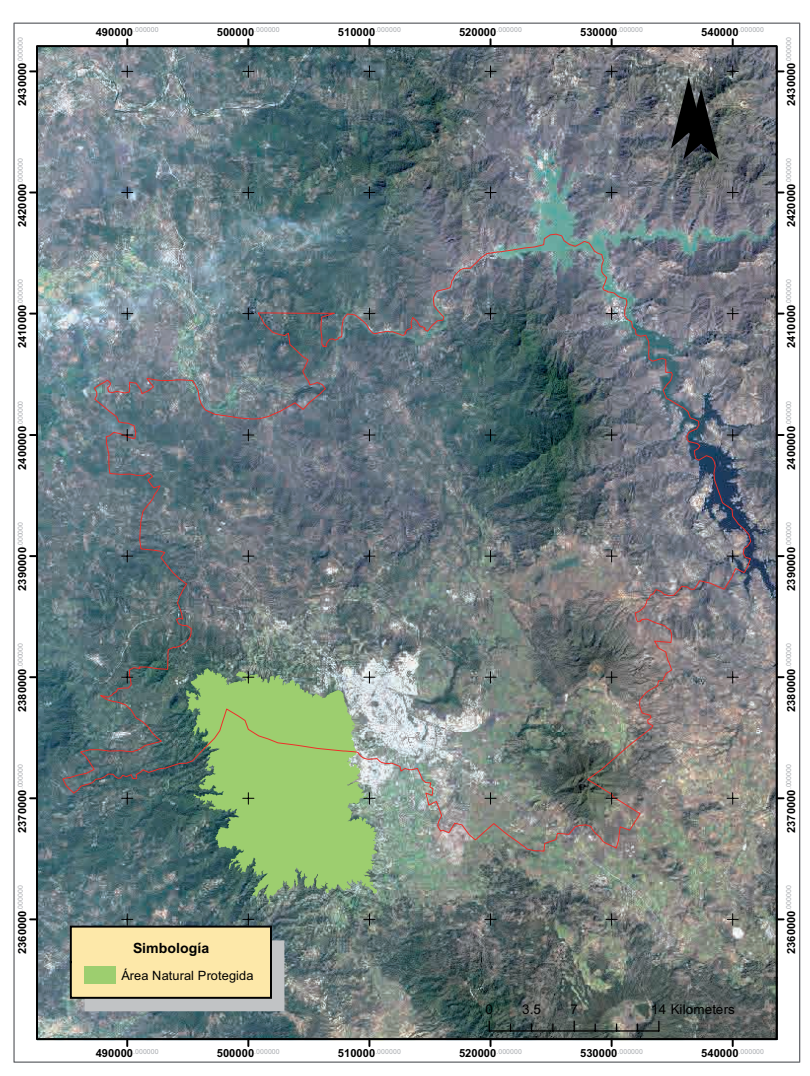

Fig. 6. Área natural protegida en la zona. Fuente: elaboración propia con información del Programa de conservación y manejo del ANP e imagen del satélite Landsat 4

Las clases secundarias "Vegetación arbórea" y "Vegetación arbustiva y herbácea" comprenden superficies alteradas de las clases anteriores y su cobertura representa el 27.88 \%. Las "Tierras agrícolas" constituyen el principal uso del suelo del municipio (32.06 \%); con áreas sembradas de caña, frutas (cítricos, aguacate, mango y ciruela), agave y hortalizas. Los "Pastizales" son el 5.07 \% del área y los asentamientos humanos el $4.04 \%$ (Fig. 8).

\section{Áreas con potencial para la instalación de un relleno sanitario}

Analizando los criterios previamente presentados, así como los señalados en la NOM-083-SEMARNAT-2003, se pudieron identificar algunos puntos potenciales para la localización de un sitio de disposición de RSU (Fig. 9).

De la superficie de 168781 ha que abarca el municipio de Tepic, sólo 9090.8 ha tienen las características adecuadas para la instalación de un relleno sanitario y 159690.4 ha se consideran no adecuadas. Es decir, el $94.6 \%$ del área en estudio

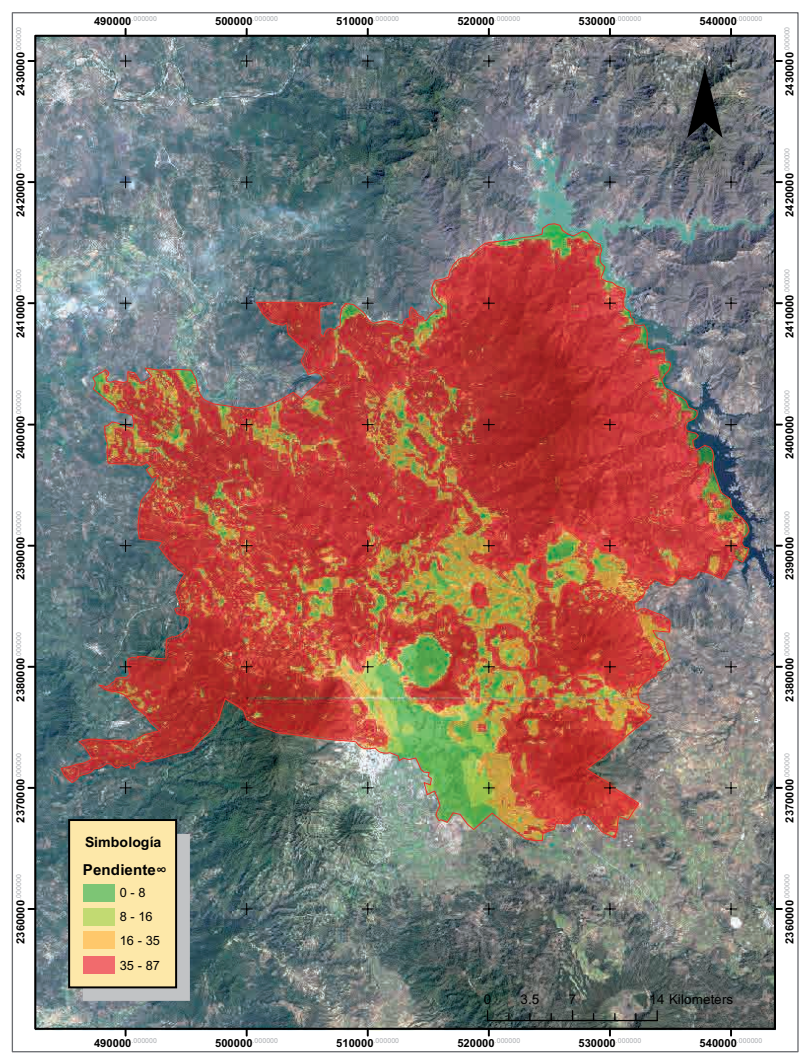

Fig. 7. Rangos de pendiente. Fuente: elaboración propia con información del INEGI (Conjunto de datos vectoriales, cartas F13 d11, d21, d31, d22, c29 y c39) e imagen del satélite Landsat 4

CUADRO IV. CLASES DE COBERTURA Y USO DEL SUELO EN EL MUNICIPIO DE TEPIC, NAYARIT

\begin{tabular}{lc}
\hline Clase de cobertura y uso del suelo & $\begin{array}{c}\text { \% de cobertura } \\
\text { en el municipio }\end{array}$ \\
\hline Bosque & 16.5 \\
Selva & 12.4 \\
Pastizal & 5.06 \\
Vegetación secundaria arbórea & 21.5 \\
Vegetación secundaria arbustiva-herbácea & 6.4 \\
Tierras agrícolas & 33.9 \\
Asentamientos humanos & 4.03 \\
\hline
\end{tabular}

presenta rasgos que se contraponen a los señalados en la NOM-083-SEMARNAT-2003, además de que su topografía, con una pendiente mayor a $35^{\circ}$, resulta desfavorable para la construcción de un relleno. A lo anterior se suma la existencia de áreas boscosas de selva, cuya protección se considera restrictiva en este análisis. Por lo tanto, sólo el $5.4 \%$ del área en estudio presenta los rasgos adecuados para la instalación de un relleno sanitario. 


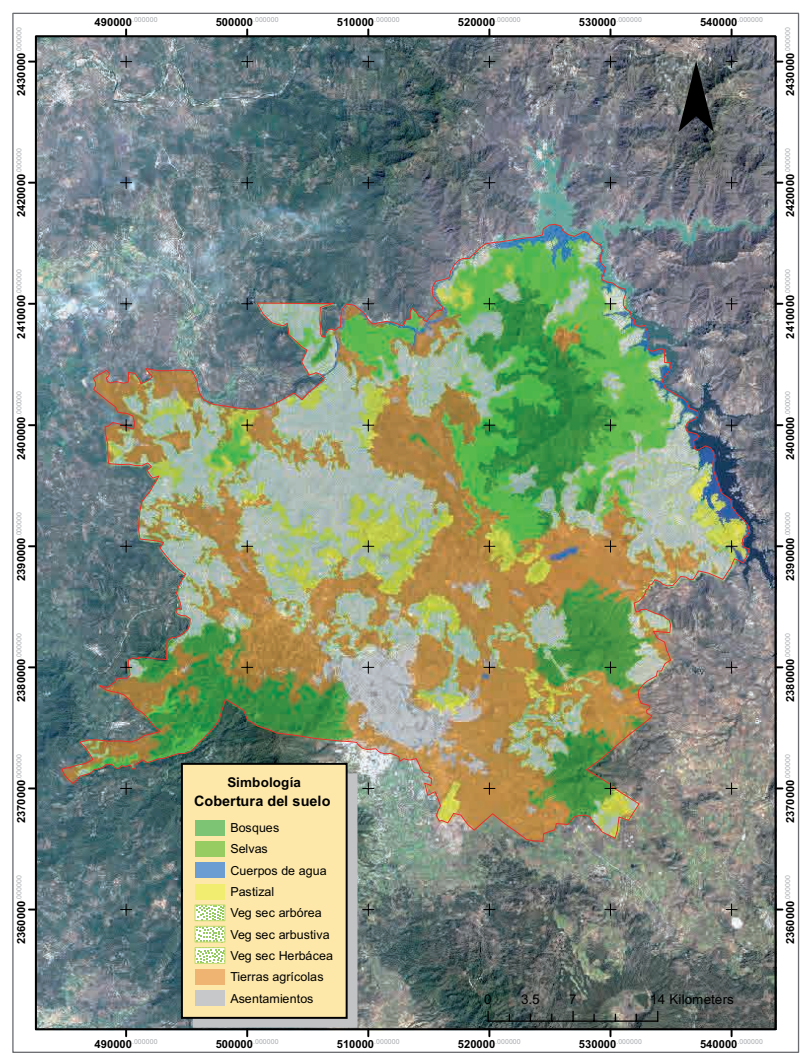

Fig. 8. Cobertura vegetal y uso del suelo. Fuente: elaboración propia con información del INEGI (Carta de uso del suelo y vegetación, serie VI) e imagen del satélite Landsat 4

\section{DISCUSIÓN}

Este trabajo ofrece un marco de evaluación para identificar el sitio potencial para la disposición final de los residuos. Se basó en criterios seleccionados de la NOM-083-SEMARNAT-2003 a los que se sumaron factores del área, reflejados en un sistema de información geográfica. El mapa generado combinó capas de información como distancia a arroyos y cuerpos de agua, áreas naturales protegidas, vegetación, pendiente, distancia a aeropuertos, centros urbanos y rurales. La identificación de áreas viables para un relleno sanitario es compleja, ya que involucra la combinación de parámetros sociales, técnicos y ambientales, así como diversas regulaciones (Şener et al. 2010).

\section{CONCLUSIÓN}

Los residuos sólidos municipales son un reto importante, no sólo por su volumen o los problemas ligados a su gestión; el desafío real se asocia con

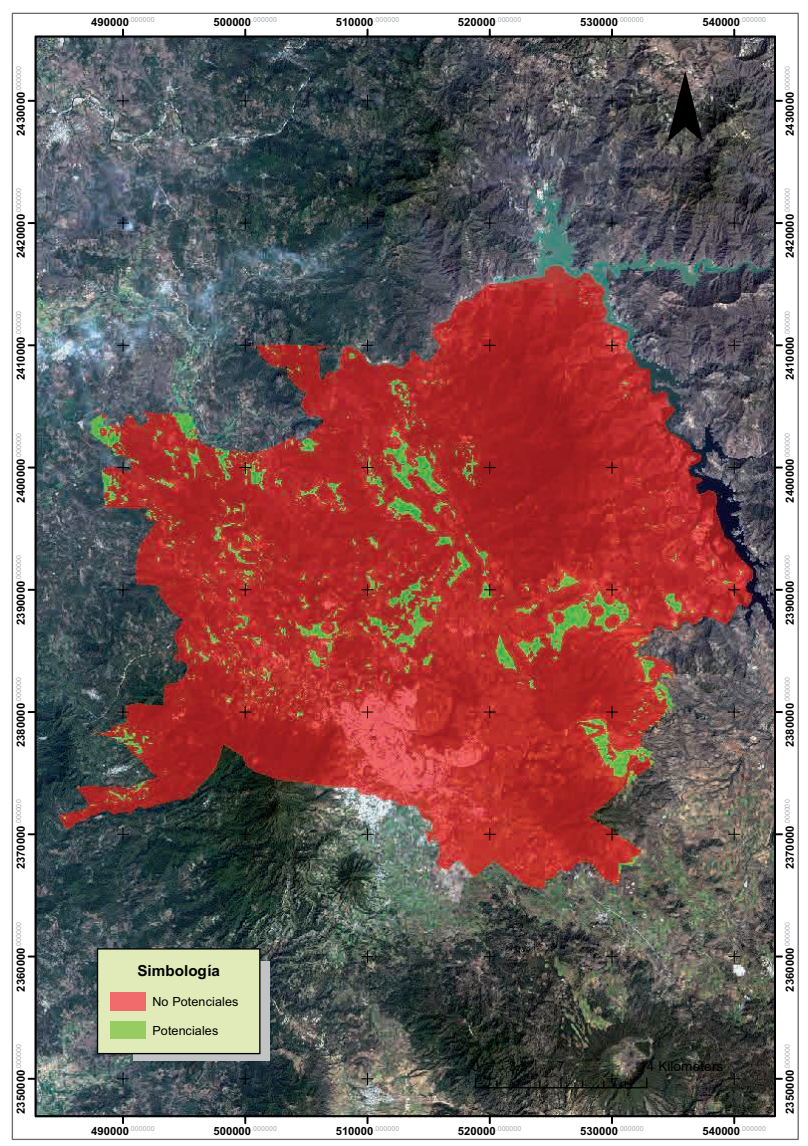

Fig. 9. Áreas potenciales para la localización de un sitio de disposición de RSU. Fuente: elaboración propia con información del INEGI e imagen del satélite Landsat 4

su disposición final: la localización de espacios o áreas del territorio adecuados para la instalación de rellenos sanitarios.

Se ha logrado identificar un $5.4 \%$ de superficie del municipio de Tepic (cerca de 9090.8 ha) con rasgos apropiados para la localización de un relleno sanitario, mientras que el $94.6 \%$ restante presenta al menos una característica que lo limita o descarta para este fin.

Se utilizaron cuatro de los criterios señalados en la NOM-083-SEMARNAT-2003: distancia mínima a centros de población, infraestructura (vías de comunicación aérea), cauces y cuerpos de agua, y áreas naturales protegidas. A éstas se sumaron dos rasgos naturales: pendiente (mayor a 30) y cobertura y uso del terreno (asociaciones de pino, encino, bosques mixtos y selvas). La inclusión de estos rasgos limita las áreas con potencial, al descartar aquellas susceptibles de deslizamientos de tierra y afectaciones a la vegetación natural, como consecuencia de las obras de ingeniería y operación asociadas al relleno sanitario. 
Este trabajo representa un acercamiento a la identificación de áreas adecuadas para la instalación de un relleno sanitario de residuos sólidos urbanos y debe completarse con estudios geológicos e hidrológicos señalados en la normativa vigente, así como estudios de impacto ambiental, riesgos, cambios de uso del suelo, consulta pública y demás instrumentos de planeación considerados en la gestión ambiental.

\section{AGRADECIMIENTOS}

Este trabajo de investigación fue financiado por el Consejo Nacional de Ciencia y Tecnología (CONACYT) dentro del proyecto de investigación aprobado como pertinente en la convocatoria Proyectos de Investigación Científica a Problemas Nacionales 2014, número de solicitud 247699.

\section{REFERENCIAS}

Bernache P.G. (2015). La gestión de los residuos sólidos: un reto para los gobiernos locales. Sociedad y Ambiente 7, 72.98. DOI: 10.31840/sya.v0i7.1592

Chabuk A., al-Ansari N., Musa Hussain H., Knutsson S. y Pusch R. (2016). Landfill site selection using Geographic Information System and Analytical Hierarchy Process: A case study Al-Hillah Qadhaa, Babylon, Iraq. Waste Manag. Res. 34 (5), 427-37.

DOI: $10.1177 / 0734242 X 16633778$

DAP (2016). Informe. Dirección de Aseo Público. H. XL Ayuntamiento de Tepic, Nayarit, México [en línea]. http://www.transparencia.tepic.gob.mx/docs/sistema/ docs/2017/20170502-1150-implan_informe_agosto-2016.pdf

El Maguiri A., Kissi B., Idrissi L. y Souabi S. (2016). Landfill site selection using GIS, remote sensing and Multicriteria Decision Analysis: Case of the City of Mohammedia, Morocco. B. Eng. Geol. Environ. 75 (3), 1301-1309. DOI: 10.1007/s10064-016-0889-z

Guiqin W., Li Q., Guoxue L. y Lijun Ch. (2009). Landfill site selection using spatial information technologies and AHP: A case study in Beijing, China. J. Environ. Manage. 90 (8): 2414-2421.

DOI: $10.1016 /$ j.jenvman.2008.12.008

Güler D. y Yomralığlu T. (2017). Alternative suitable landfill site selection using Analytic Hierarchy Process and Geographic Information Systems: A case study in Istanbul. Environ. Earth. Sci. 76 (20), 678.

DOI: $10.1007 / \mathrm{s} 12665-017-7039-1$

INEGI (2016). Anuario Estadístico y Geográfico de Nayarit 2016. Instituto Nacional de Estadística y
Geografía, México [en línea]. http://seplan.gob.mx/ siegen/ds/Anuario\%20Estadistico/ANUARIO\%20 ESTADISTICO\%202016.pdf

Khan D. y Samadder S.R. (2014). Municipal solid waste management using geographical Information System aided methods: A mini review. Waste Manage. Res. 32 (11), 1049-1062. DOI: 10.1177/0734242X14554644

Malczewski J. (2004). GIS-based land-use suitability analysis: A critical overview. Prog. Plann. 62 (1), 3-65. DOI: $10.1016 /$ j.progress.2003.09.002

Sadek S., el-Fadel M. y Freiha F. (2006). Compliance factors within a GIS-based framework for landfill siting. Int. J. Environ. Stud. 63 (1), 71-86. DOI: $10.1080 / 00207230600562213$

SEDERMA (2008). Programa de Conservación y Manejo del Área Natural Protegida Reserva de la Biosfera Estatal Sierra de San Juan. Secretaría del Medio Ambiente del estado de Nayarit. Periódico Oficial del Gobierno del Estado de Nayarit, 13 de diciembre.

Şener Ş., Şener E., Nas B. y Karagüzel R. (2010). Combining AHP with GIS for landfill site selection: A case study in the Lake Beysehir catchment area (Konya, Turkey). Waste Manage. 30 (11), 2037-2046.

DOI: 10.1016/j.wasman.2010.05.024

Segura, G., Badilla, E. y Obando, L. (2011). Susceptibilidad al deslizamiento en el corredor Siquirres-Turrialba. Revista Geológica de America Central, 45,101-121.

SEMARNAT (2004). NOM-083-SEMARNAT-2003. Especificaciones de protección ambiental para la selección del sitio, diseño, construcción, operación, monitoreo, clausura y obras complementarias de un sitio de disposición final de residuos sólidos urbanas y de manejo especial. Secretaría de Medio Ambiente y Recursos Naturales, Diario Oficial de la Federación, 20 de octubre.

UNDESA (2014). World urbanization prospects: The 2014 revision. Population Division, United Nations Department of Economic and Social Affairs [en línea]. https://population.un.org/wup/Publications/Files/ WUP2014-Report.pdf

USGS (2018). Global Visualization Viewer (GloVis). United States Geological Survey [en línea].: http:// glovis.usgs.gov

Vergara S.E. y Tchobanoglous G. (2012). 37 annual review of environment and resources municipal solid waste and the environment: A global perspective [en línea]. http://www.annualreviews.org/doi/10.1146/annurevenviron-050511-122532

Zamorano M., Molero E., Hurtado A., Grindlay A. y Ramos A. (2008). Evaluation of a municipal landfill site in southern Spain with GIS-Aided methodology. J. Hazard. Mater. 160 (2-3), 473-481. DOI: $10.1016 /$ j.jhazmat.2008.03.023 